Supplementary Information for

\title{
Enzyme-/Redox- Responsive Mesoporous Silica Nanoparticles Based on Functionalized Dopamine as Nanocarriers for Cancer Therapy
}

Dandan Zhu ${ }^{\dagger}$, Chunlin $\mathrm{Hu}^{\dagger}$, Yuan Liu ${ }^{\dagger}$, Feng Chen ${ }^{\dagger}$, Zhen Zheng ${ }^{\dagger}$, Xinling Wang*†

†School of Chemistry and Chemical Technology, State Key Laboratory of Metal Matrix Composites, Shanghai Jiao Tong University, Shanghai 200240 China

*Correspondence to: xlwang@sjtu.edu.cn

\section{Contents}

Figure S1. ${ }^{1} \mathrm{H}$ NMR spectrum of (Boc) ${ }_{2}-\mathrm{Cy}-\mathrm{DA}$.

Table S1. The element analysis results of $(\mathrm{Boc})_{2}$-Cystine-dopamine

Figure S2. NMR spectra of Cy-DA: (a) ${ }^{1} \mathrm{H}$ NRM, (b) ${ }^{13} \mathrm{C}$ NMR.

Figure S3. TOF-MS spectrum of Cy-DA.

Scheme S1. The propose cross-link of poly-Cy-DA in self- polymerization process.

Figure S4. (a) EDX spectrum of Cy-DA-MSNs. (b) Mapping results of Si. (c) Mapping results of S. (d) EDX spectrum of MSNs.

Figure S5. Water contact angle of glass and poly-Cy-DA modified glass.

Figure S6. The fluorescence microscope images of cellular uptake of DOX@poly-Cy-DA-MSNs nanoparticles $(10 \mu \mathrm{g} / \mathrm{mL})$ and their intracellular drug release behavior after incubation with Hela cells for 6,12 , and $24 \mathrm{~h}$. 


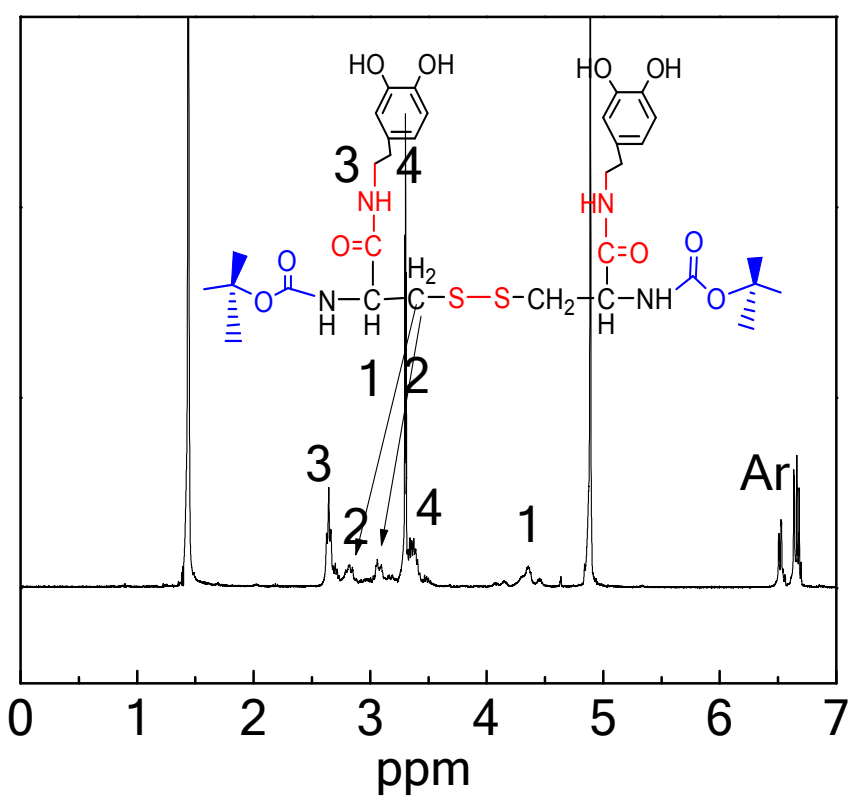

Figure S1. ${ }^{1} \mathrm{H}$ NMR spectrum of $(\mathrm{Boc})_{2}-\mathrm{Cy}-\mathrm{DA}$.

Table S1. The element analysis results of (Boc) $)_{2}$-Cystine-dopamine

\begin{tabular}{ccc|ccc}
\hline \multicolumn{2}{c|}{ Test results (Percent, \%) } & \multicolumn{3}{|c}{ Theoretic results (Percent, \%) } \\
\hline$(\mathrm{N} \%)$ & $(\mathrm{C} \%)$ & $(\mathrm{S} \%)$ & $(\mathrm{N} \%)$ & $(\mathrm{C} \%)$ & $(\mathrm{S} \%)$ \\
\hline $7.73 \%$ & $50.55 \%$ & 9.88 & 7.88 & 54.07 & 9.02 \\
\hline
\end{tabular}

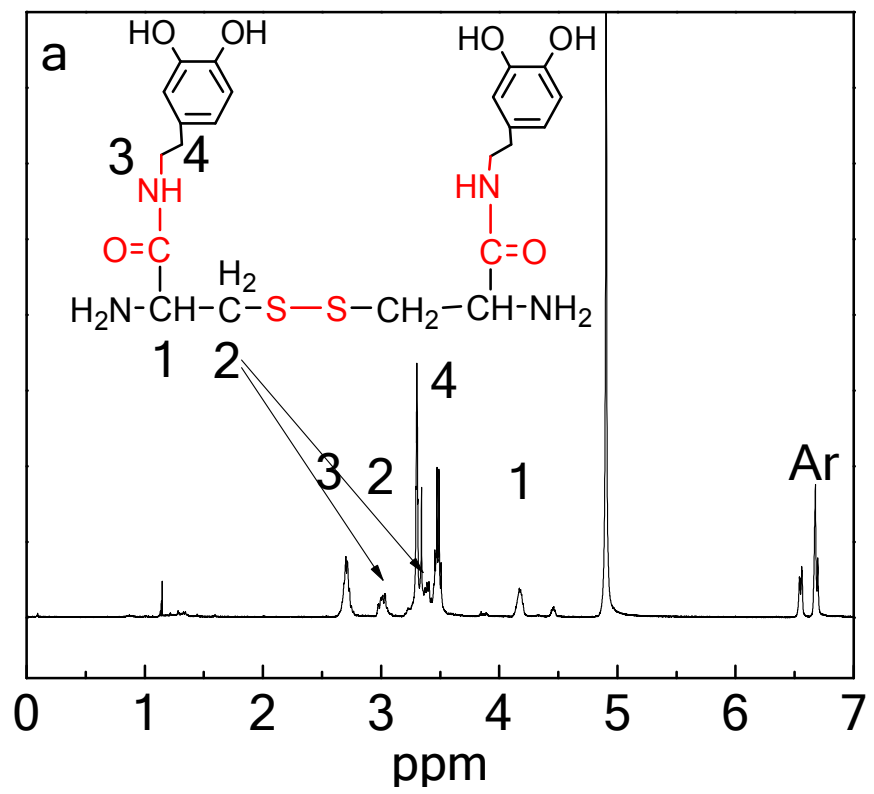




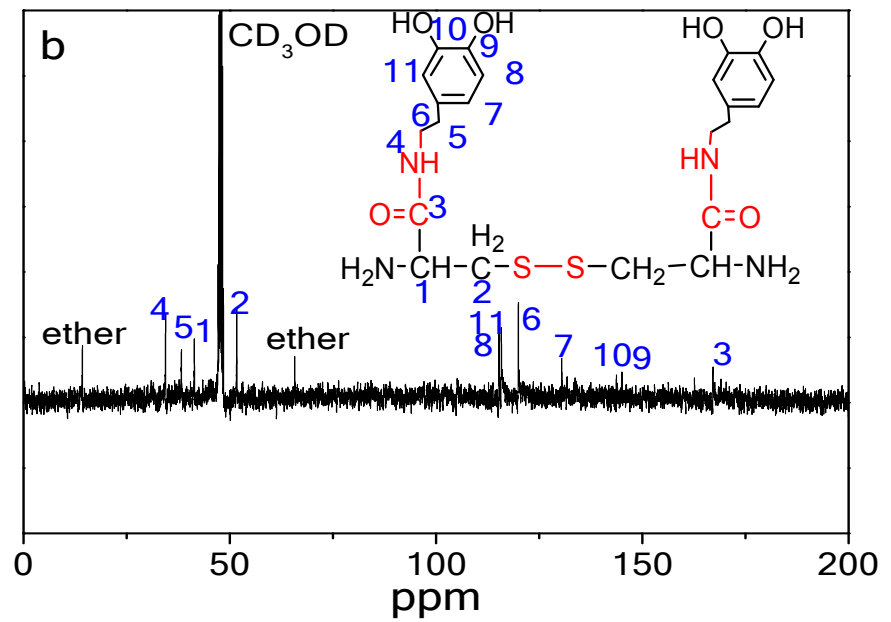

Figure S2. NMR spectra of Cy-DA: (a) ${ }^{1} \mathrm{H}$ NRM, (b) ${ }^{13} \mathrm{C}$ NMR.

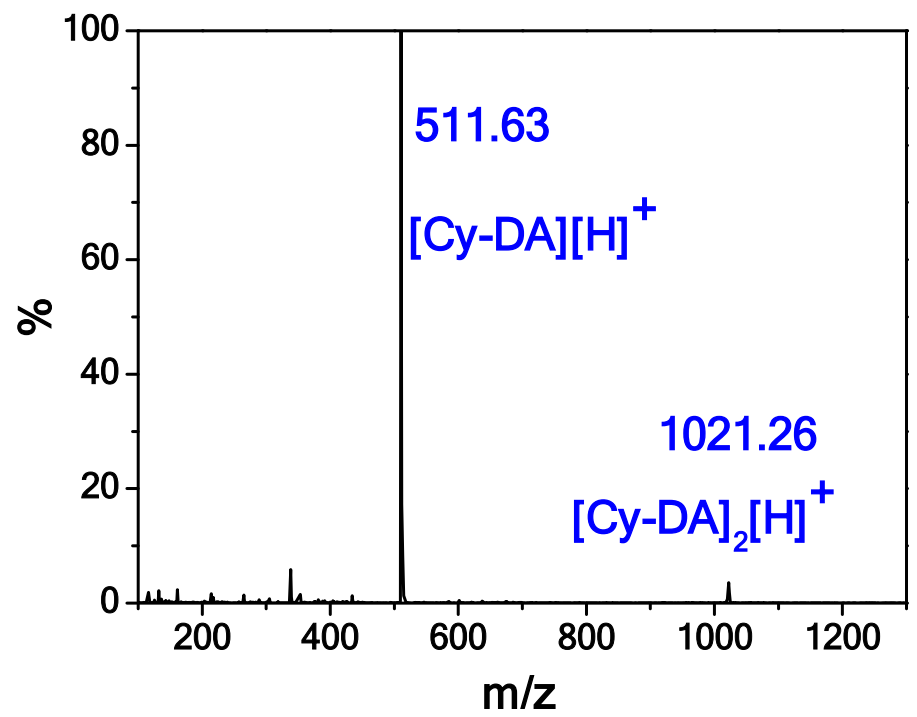

Figure S3. TOF-MS spectrum of Cy-DA.

\section{The propose Cross-link of poly-Cy-DA in self-polymerization process.}

The catechol was easy to be oxidized and formed quinone structure. The primary roles of adhesive bonding and cross-link formation can be assigned to the reverse dismutation reaction between catechol ando-quinone form of poly-Cy-DA and other catechol compounds molecule. Then the poly-Cy-DA formed a thin polymer layer on the glass surfaces and introduced polar groups such as carboxyl and amine onto the membranes. It was supposed that the cross-link reaction between poly-Cy-DA molecules followed the route described in the literatures ${ }^{45}$. The possible reaction mechanism of poly-Cy-DA was shown in Scheme S1. However, the exact polymerization and interaction mechanism of the adhesive poly-Cy-DA layer was kept unknown so far ${ }^{1-3}$. 

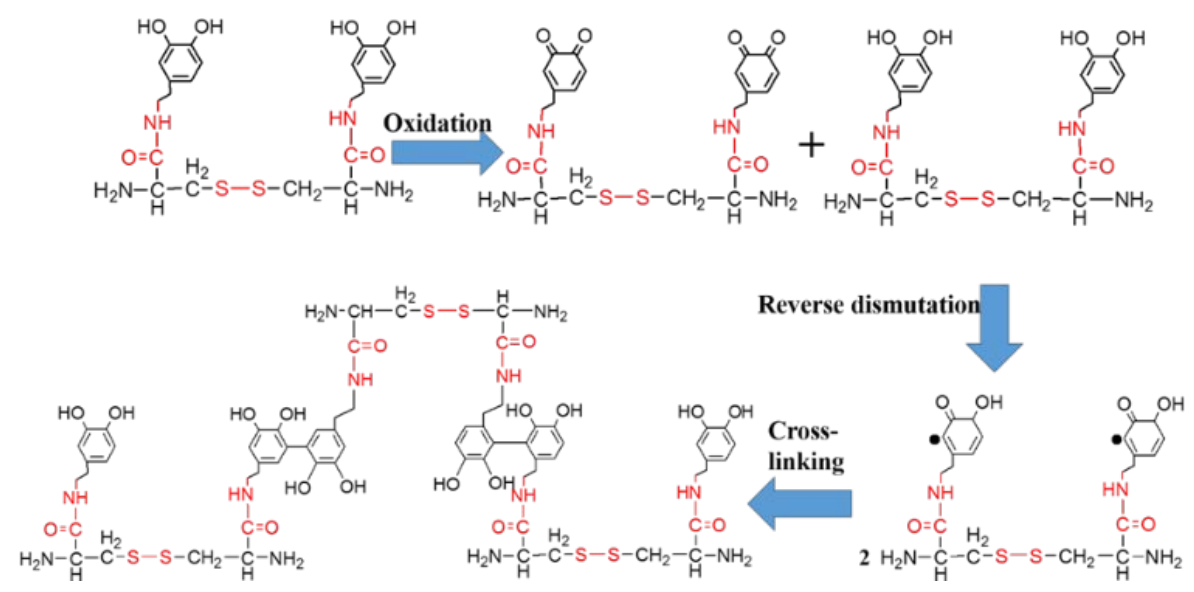

Scheme S1. The propose Cross-link of poly-Cy-DA in self- polymerization process.

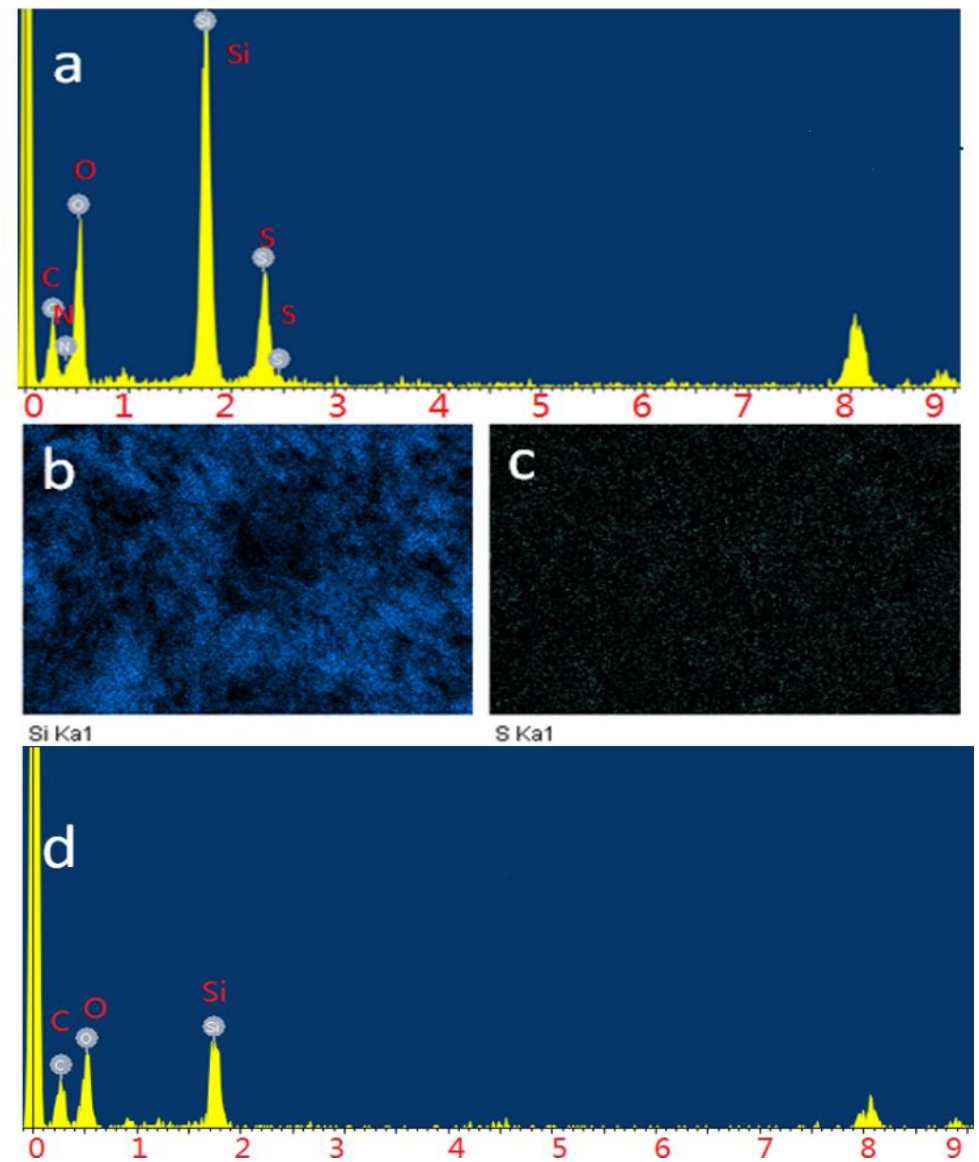

Figure S4. (a) EDX spectrum of Cy-DA-MSNs. (b) Mapping results of Si. (c) Mapping results of S. (d) EDX spectrum of MSNs. 


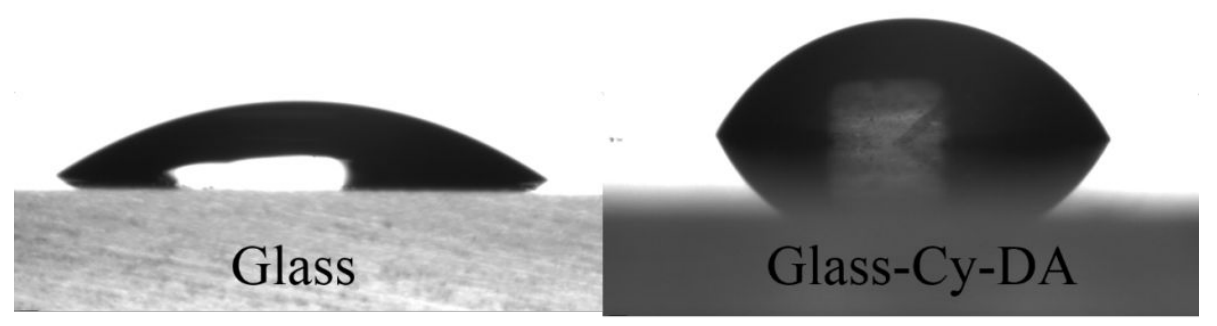

Figure S5. Water contact angle of glass and poly-Cy-DA modified glass.

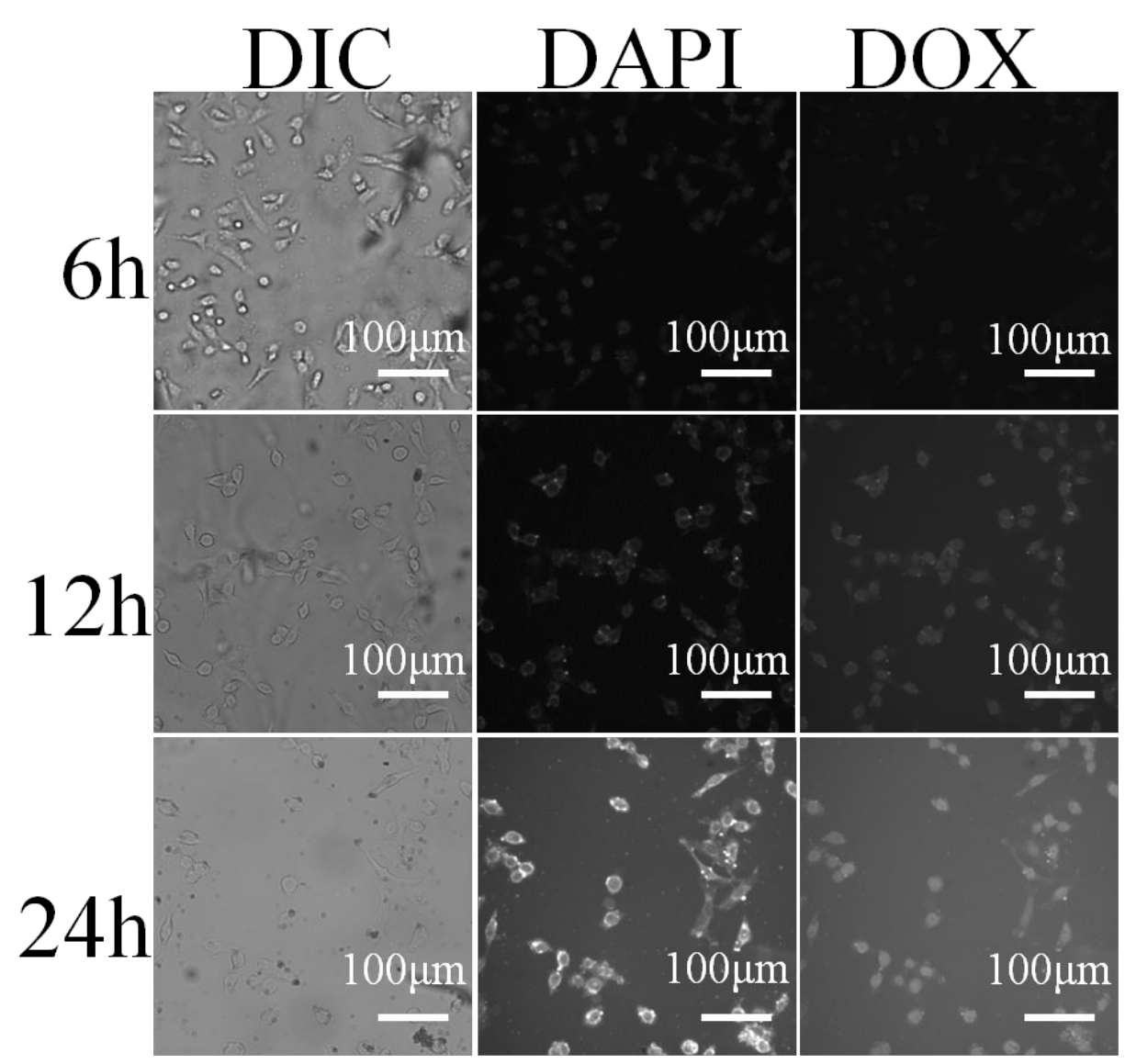

Figure S6. The fluorescence microscope images of cellular uptake of DOX@poly-Cy-DA-MSNs nanoparticles $(10 \mu \mathrm{g} / \mathrm{mL})$ and their intracellular drug release behavior after incubation with Hela cells for 6, 12, and $24 \mathrm{~h}$. (scale bar: $100 \mu \mathrm{m})$

\section{Reference}

(1) Lee, H.; Scherer, N. F.; Messersmith, P. B. Single-Molecule Mechanics of Mussel Adhesion. Proc. Natl. Acad. Sci. USA 2006, 103, 12999-13003. 
(2) Burzio, L. A.; Waite, J. H.; Cross-Linking in Adhesive Quinoproteins: Studies with Model Decapeptides. Biochemistry 2000, 39, 11147-11153.

(3) Van, der Leeden M. C. Are Conformational Changes, Induced by Osmotic Pressure Variations, the Underlying Mechanism of Controlling the Adhesive Activity of Mussel Adhesive Proteins? Langmuir 2005, 21, 11373-11379. 